\section{Associação entre insegurança alimentar e níveis de hemoglobina e retinol em crianças assistidas pelo Sistema Único de Saúde no Município do Rio de Janeiro, Brasil}

\author{
Association between food insecurity and \\ hemoglobin and retinol levels in children treated \\ in the Brazilian Unified National Health System in \\ the city of Rio de Janeiro, Brazil
}

\section{Asociación entre inseguridad alimentaria y niveles de hemoglobina y retinol en niños asistidos por el Sistema Único de Salud en el municipio de Río de Janeiro, Brasil}

Letícia Barroso Vertulli Carneiro 1 Inês Rugani Ribeiro de Castro 2 Leidjaira Lopes Juvanhol 3

Fabio da Silva Gomes 4

Letícia de Oliveira Cardoso 1

\title{
Resumo
}

Neste trabalho foi analisada a associação entre insegurança alimentar e níveis de hemoglobina e retinol em crianças de 6 a 59 meses de idade. Trata-se de um estudo seccional, realizado em 2014, com amostra representativa da população de crianças nessa faixa etária, atendidas em unidades básicas de saúde do Município do Rio de Janeiro, Brasil. Para a análise dos níveis de insegurança alimentar foi utilizada a Escala Brasileira de Insegurança Alimentar $e$, para a determinação de hemoglobina e de retinol sérico, foi realizada a punção venosa. A associação entre as variáveis foi avaliada por intermédio de modelos de regressão quantílica. Do total de crianças estudadas, 40,3\% apresentavam insegurança alimentar e as prevalências de anemia e de deficiência de vitamina A foram 13,7\% e 13\%, respectivamente. Os resultados do estudo revelaram associação inversa, estatisticamente significativa, entre insegurança alimentar leve e níveis de retinol. Para os demais níveis de insegurança alimentar (moderada e grave), os resultados também sugerem a presença de associação inversa para hemoglobina e, quanto aos níveis de retinol, as estimativas pontuais parecem menores em crianças com insegurança alimentar grave, entretanto, estas estimativas não foram estatisticamente significativas. Esses resultados sugerem que a insegurança alimentar pode estar associada com carências de micronutrientes em crianças menores de 5 anos.

Segurança Alimentar e Nutricional; Anemia; Deficiência de Vitamina A; Criança

\author{
Correspondência \\ L. B. V. Carneiro \\ Fundação Oswaldo Cruz. \\ Rua Leopoldo Bulhões 1480, Rio de Janeiro, RJ \\ 21041-210, Brasil. \\ leticiavertulli@yahoo.com.br \\ 1 Fundação Oswaldo Cruz, Rio de Janeiro, Brasil. \\ 2 Universidade do Estado do Rio de Janeiro, Rio de Janeiro, \\ Brasil. \\ 3 Universidade Federal de Viçosa, Viçosa, Brasil. \\ 4 Pan American Health Organization, Washington DC, U.S.A.
}




\section{Introdução}

O relatório de insegurança alimentar no mundo, publicado pela Organização das Nações Unidas para Agricultura e Alimentação (FAO) em 2014, revelou que a subalimentação, marcador utilizado pela agência para dimensionar e acompanhar a fome em âmbito internacional, caiu de 23,4\% para 13,5\% entre 1990 e 2013 nos países em desenvolvimento. No Brasil, esse indicador atingiu valores abaixo de $5 \%$, o que significa que o país vinha, no decorrer das últimas décadas, superando o problema da fome 1.

Resultados da Pesquisa Nacional de Demografia e Saúde (PNDS) conduzida em 2006, que avaliou a segurança alimentar (definida brevemente como acesso regular a alimentos de qualidade, em quantidade suficiente, sem comprometer o acesso a outras necessidades essenciais) 2 em domicílios brasileiros com mulheres em idade fértil, mostraram que a insegurança alimentar estava presente em 45,5\% dos domicílios com crianças menores de cinco anos 3,4. Resultados de dois inquéritos nacionais em anos subsequentes mostraram uma queda da prevalência de insegurança alimentar domiciliar, passando de 30,2\% em 20095 para 22,6\% em 20136

Apesar dos avanços nesses indicadores nutricionais, o Brasil agora vem vivenciando importantes consequências de uma alimentação inadequada devido, principalmente, à diminuição do consumo de alimentos in natura e ao aumento de produtos ultraprocessados 7,8,9,10. Em um estudo realizado com uma amostra da população brasileira com 10 anos ou mais de idade, o aumento da participação de alimentos ultraprocessados na dieta esteve inversamente associado ao teor de 11 micronutrientes na dieta, inclusive o ferro ${ }^{11}$.

Paralelamente, os únicos dados nacionais disponíveis sobre deficiência de micronutrientes entre crianças menores de cinco anos de idade, produzidos em 2006, apontavam para a persistência de índices elevados de anemia e deficiência de vitamina A, com prevalências de 20,9 e 17,4\%, respectivamente ${ }^{4}$. A baixa escolaridade materna, a presença de infecções recorrentes, o status materno de micronutrientes, a dieta e a absorção inadequada 12,13,14,15,16 são alguns dos principais determinantes da deficiência de vitamina A e de anemia. Entretanto, estudos sobre a associação entre insegurança alimentar e esses agravos são escassos 17,18,19,20,21,22, especialmente no contexto contemporâneo. Além disso, as evidências existentes são inconclusivas, pois foram geradas, em sua maioria, em locais de baixo nível socioeconômico, como na Região Nordeste do país, e usaram métodos de coleta de sangue simplificados que, em geral, são mais imprecisos do que aqueles considerados métodos de referência 23,24,25. Outro aspecto importante a ser destacado é que a totalidade dos estudos acerca dessa relação utilizam estratégias analíticas convencionais, que não consideram a possibilidade da existência de associações não lineares entre a insegurança alimentar e os níveis de ferro e vitamina A.

Reconhecendo a importância do status desses nutrientes 26,27,28 e da segurança alimentar para a saúde infantil $8 \mathrm{e}$, ainda, supondo que as crianças em situação de insegurança alimentar domiciliar poderiam estar mais vulneráveis à deficiência de nutrientes, o objetivo do estudo foi analisar a associação entre insegurança alimentar e níveis de hemoglobina e retinol sérico em crianças de 6 a 59 meses de idade, assistidas em unidades básicas de saúde (UBS) no Município do Rio de Janeiro, Brasil. Este trabalho pretende contribuir para a compreensão do fenômeno da insegurança alimentar e nutricional e sua relação com as deficiências de ferro e de vitamina A entre crianças de uma grande metrópole brasileira, e em um cenário nacional de menores prevalências destes eventos.

\section{Métodos}

\section{Desenho e população de estudo}

Trata-se de estudo transversal inserido em uma pesquisa maior denominada Anemia e Deficiência de Vitamina A em Pré-escolares: Magnitude em uma Grande Metrópole e Validação de Métodos Diagnósticos.

Foi realizado com uma amostra probabilística composta por crianças com idades entre 6 e 59 meses, assistidas nas UBS do Sistema Único de Saúde (SUS) no Município do Rio de Janeiro. Essa população foi escolhida devido ao fato de as políticas de controle da anemia e da deficiência de vitamina A preconizadas pelo Ministério da Saúde serem dirigidas a esse público e operacionalizadas na rede do SUS. 
A seleção das crianças ocorreu em dois estágios; no primeiro, as UBS foram estratificadas segundo: (a) tamanho, definido pelo número de atendimentos de crianças menores de cinco anos; (b) número de crianças dessa faixa etária beneficiárias do programa Bolsa Família; e (c) proporção de crianças cadastradas nas unidades em cada faixa etária (entre 6 e 23 meses, e entre 24 e 59 meses). Em seguida à estratificação, 33 UBS foram selecionadas com probabilidade proporcional ao tamanho do estrato ao qual pertenciam. A possibilidade de seleção das unidades amostrais (crianças) equivaleu à perspectiva de seleção da UBS multiplicada pela probabilidade de seleção da criança, dado que a criança pertencia à UBS selecionada.

Para o segundo estágio de seleção, foi disponibilizada por cada UBS selecionada uma listagem com data de nascimento e telefone de todas as crianças menores de cinco anos cadastradas. Em seguida, os nomes de todas as crianças foram ordenados aleatoriamente e a listagem aleatória de cada UBS foi percorrida até que fossem agendadas 44 crianças por UBS (número baseado no estudo piloto para que alcançássemos o número necessário de crianças). Foram excluídas as crianças portadoras de doença falciforme ou de qualquer hepatopatia.

Os parâmetros usados para o cálculo do tamanho amostral foram os seguintes: estimativa de 252 mil crianças na faixa etária de interesse no Município do Rio de Janeiro no ano de 2012; estimativa de prevalência de anemia de $60,2 \%$, valor adotado com base em revisão sistemática de estudos realizados em serviços de saúde 29; estimativa de prevalência de deficiência de vitamina A de $22 \%$, valor adotado baseando-se nos resultados da PNDS-2006 para a Região Sudeste do Brasil 4; precisão das estimativas de 5\%; estimativa de 1,6 para o efeito de desenho amostral. Com esses parâmetros, chegou-se a um número amostral de 588 crianças. Das 536 avaliadas na pesquisa maior, foram aqui analisadas as informações de 519 crianças, sendo excluídas aquelas que não completaram o questionário ou que não possuíam as medidas bioquímicas disponíveis $(\mathrm{n}=17)$.

\section{Coleta de dados}

A coleta de dados foi realizada entre os meses de julho e dezembro de 2014. Os responsáveis que aceitaram participar do estudo compareceram às UBS e responderam a um questionário contendo perguntas sobre características sociodemográficas, situação de saúde da criança e insegurança alimentar domiciliar.

Essa insegurança foi avaliada segundo a Escala Brasileira de Insegurança Alimentar (EBIA), validada por Pérez-Escamilla et al. 30. Utilizou-se a categorização proposta pelos pesquisadores que validaram a EBIA para o Brasil e que tem sido usada em estudos de base populacional, como a Pesquisa Nacional por Amostras de Domicílios (PNAD-2009) 4,6. O instrumento tem 14 perguntas referentes aos últimos três meses, classificando o domicílio em situação de segurança alimentar (escore zero), insegurança alimentar leve (1-5 pontos), insegurança alimentar moderada (6-9 pontos) e insegurança alimentar grave (11-14 pontos). A escala aborda desde a preocupação com a falta de alimentos até dias em que os moradores ficaram efetivamente sem comer por falta de dinheiro e, também, perguntas específicas para moradores menores de 18 anos de idade.

A coleta de sangue foi realizada por punção venosa por técnicos em patologia clínica treinados e seguiu as normas de boas práticas de pós-coleta recomendadas para evitar a degradação do retinol por exposição a raios ultravioletas 31 . O processamento das amostras de sangue e a dosagem de retinol e hemoglobina foram realizados em laboratório especializado. Para o hemograma completo, o sangue foi submetido à contagem automatizada em aparelho hematológico, marca Coulter T890 (Beckman Coulter Inc., Estados Unidos). O retinol sérico foi dosado pelo método Cromatografia Líquida de Alta Eficiência, utilizando metodologia de extração adaptada de Hess et al. 32 em condições cromatográficas propostas por Craft et al. 33. A dosagem de proteína $\mathrm{C}$ reativa (PCR, analisada como covariável) foi realizada por método de Turbidimetria de alta sensibilidade por leitura em espectrofotômetro a 540nm.

Todos os procedimentos de coleta de dados foram padronizados segundo um manual de campo e diferentes protocolos para cada atividade da pesquisa. Antes do início da coleta de dados, foram realizados pré-testes dos instrumentos a serem utilizados no trabalho e um estudo piloto em uma UBS do município que não foi sorteada para entrar na pesquisa. 


\section{Análise dos dados}

Os desfechos de interesse foram: concentração sérica do retinol e concentração de hemoglobina. Para a descrição da população foram estimadas as prevalências de anemia (hemoglobina menor que $11 \mathrm{mg} / \mathrm{dL}$ ) e deficiência de vitamina A (retinol menor que 0,70 $\mu \mathrm{mol} / \mathrm{L}$ ) 34 .

As análises da associação entre a insegurança alimentar e os desfechos foram realizadas utilizando-se modelos de regressão quantílica, que representam uma extensão dos modelos lineares para diferentes quantis amostrais da distribuição do desfecho estudado (níveis de hemoglobina e de retinol). Os modelos lineares estimam os coeficientes para a média da variável dependente, e a regressão quantílica produz estas estimativas para qualquer quantil da distribuição da variável dependente 35 . Dessa forma, esse tipo de análise possibilitou observar a associação entre a insegurança alimentar e diferentes quantis da distribuição de hemoglobina e retinol. Além disso, a regressão quantílica é um método bastante robusto e pouco sensível a outliers, e nenhuma suposição é feita em relação à distribuição da variável dependente. Assim, pode ser bastante apropriada para analisar variáveis que não têm uma distribuição normal, ou em situações em que a relação entre as variáveis de exposição e desfecho não seja linear 36,37 . Neste estudo, não eram esperadas mudanças na direção da curva, ascendente ou descendente, mas a linha toda de estimativas mais baixa na insegurança grave em relação à leve. Como uma relação dose-resposta: maiores reduções nos coeficientes na medida em que o nível de insegurança aumenta. Uma vez que a categoria de referência é a segurança alimentar, era esperado que crianças com insegurança alimentar grave tivessem estimativas mais baixas de retinol e hemoglobina do que aquelas com insegurança alimentar leve, ou seja, esperava-se ambas abaixo da linha pontilhada, porém, a grave é ainda mais baixa que a leve.

Os modelos de regressão foram inicialmente ajustados pelas seguintes covariáveis: faixa etária da criança ( $<2$ ou $\geq 2$ anos); nível de escolaridade materna (Ensino Fundamental incompleto, completo, Ensino Médio e Ensino Superior); nível de renda familiar (<0,5; 0,5-0,9; 1-1,9; 2-2,9; 3 ou mais salários mínimos por domicílio); recebimento de benefícios do governo (sim ou não); e concentração sérica de PCR. Essas covariáveis foram selecionadas por serem potenciais confundidoras da associação de interesse em estudos que avaliaram determinantes das variáveis analisadas no presente artigo 12,13,14,15,16.

Os modelos finais foram construídos com as variáveis de ajuste que, após a inclusão nos mesmos, mantiveram o valor de p menor que 0,05. Dessa forma, permaneceram no modelo de hemoglobina a faixa etária da criança e, no caso do retinol, a PCR e a escolaridade materna.

Em todas as análises foram considerados os estratos amostrais e os respectivos fatores de expansão. Os coeficientes da regressão foram estimados com intervalos regulares, a cada cinco quantis, do 5o ao 95o, e expressam a diferença nos níveis de retinol e hemoglobina (em $\mu \mathrm{mol} / \mathrm{L}$ e $\mathrm{mg} / \mathrm{dL}$, respectivamente) para as categorias de insegurança alimentar em comparação à categoria de referência (segurança alimentar), considerando os diferentes quantis da distribuição dos desfechos. O programa empregado na análise dos dados foi o R versão 3.2.3 (http://www.r-project.org).

A pesquisa maior na qual o estudo está inserido foi aprovada pelo Comitê de Ética em Pesquisa com Seres Humanos da Secretaria Municipal de Saúde do Rio de Janeiro (no 93/13).

\section{Resultados}

A prevalência de insegurança alimentar foi de $40 \%$ e a forma leve estava presente em 33\% das crianças. Com relação à escolaridade materna, 20\% das mães não haviam completado o Ensino Fundamental e 42\% tinham concluído o Ensino Médio. A maior parte das famílias (61\%) recebia menos de dois salários mínimos por mês. As deficiências de micronutrientes (ferro e vitamina A) atingiam em torno de $13 \%$ das crianças (Tabela 1).

Apesar de as médias de hemoglobina e retinol não apresentarem uma relação linear com os níveis de insegurança alimentar, parece existir uma tendência de redução das mesmas conforme aumenta a gravidade da insegurança alimentar. Com relação à escolaridade materna, os níveis de retinol aumentaram de forma significativa com o aumento da escolaridade. Ao comparar crianças de 6 a 23 meses com as maiores (de dois a cinco anos) a média de hemoglobina foi menor entre as mais novas (Tabela 2). 


\section{Tabela 1}

Caracterização das crianças de 6 a 59 meses assistidas em unidades básicas de saúde, Município do Rio de Janeiro, Brasil, 2014.

\begin{tabular}{|c|c|}
\hline Variáveis sociodemográficas e de saúde & $\%$ \\
\hline \multicolumn{2}{|l|}{ Sexo } \\
\hline Feminino & 49,8 \\
\hline Masculino & 50,1 \\
\hline \multicolumn{2}{|l|}{ Faixa etária (meses) } \\
\hline $6-23$ & 31,0 \\
\hline $24-59$ & 69,0 \\
\hline \multicolumn{2}{|l|}{ Nível de insegurança alimentar } \\
\hline Segurança alimentar & 59,7 \\
\hline Insegurança leve & 33,3 \\
\hline Insegurança moderada & 3,9 \\
\hline Insegurança grave & 3,1 \\
\hline \multicolumn{2}{|l|}{ Deficiências de micronutrientes * } \\
\hline Anemia & 13,7 \\
\hline Deficiência de vitamina A & 13,0 \\
\hline \multicolumn{2}{|l|}{ Escolaridade materna } \\
\hline Ensino Fundamental incompleto & 20,4 \\
\hline Ensino Fundamental completo & 34,2 \\
\hline Ensino Médio & 42,0 \\
\hline Ensino Superior & 3,32 \\
\hline \multicolumn{2}{|l|}{ Renda familiar (em salários mínimos) } \\
\hline$<0,5$ & 6,0 \\
\hline $0,5-0,9$ & 9,0 \\
\hline $1-1,9$ & 46,1 \\
\hline $2-2,9$ & 23,2 \\
\hline 3 ou mais & 15,7 \\
\hline \multicolumn{2}{|l|}{ Recebe benefícios do governo } \\
\hline $\operatorname{Sim}$ & 37,2 \\
\hline Não & 62,8 \\
\hline
\end{tabular}

* Ponto de corte para a classificação de deficiência de vitamina A: retinol sérico $<0,70 \mu \mathrm{mol} / \mathrm{L}$. Ponto de corte para classificação de anemia: concentração de hemoglobina $<11 \mathrm{mg} / \mathrm{dL} 34$.

As associações entre insegurança alimentar e os níveis de retinol sérico e de hemoglobina, estimadas por meio de regressão quantílica, estão apresentadas na Figura 1. É possível observar as diferenças nos coeficientes para os diferentes quantis da distribuição de retinol e de hemoglobina segundo os níveis de insegurança alimentar comparados à categoria de referência (segurança alimentar). Com relação ao retinol, crianças com insegurança alimentar leve possuíam níveis mais baixos deste micronutriente quando comparadas àquelas com segurança alimentar. Os coeficientes foram significativos do quantil 35 ao 90, com o maior valor observado no quantil 85, em que a insegurança alimentar leve foi associada a uma redução de $0,15 \mu \mathrm{mol} / \mathrm{L}$ nos níveis de retinol. Crianças com insegurança alimentar grave também apresentaram níveis mais baixos de retinol considerando-se as estimativas pontuais, porém, os IC95\% (intervalo de 95\% de confiança) dos coeficientes incluíram o valor zero em todos os quantis da distribuição.

Observaram-se, ainda, menores níveis de hemoglobina nas categorias de insegurança alimentar moderada e grave. Entretanto, essas diferenças não foram estatisticamente significativas, uma vez que os IC95\% dos coeficientes (representados pela área cinza do gráfico) incluem a nulidade (Figura 1).

Foram realizadas análises adicionais para exame das associações com os desfechos na forma binária (presença de anemia e de hipovitaminose A) e com a variável de exposição em diferentes 


\section{Tabela 2}

Média de hemoglobina e de retinol sérico segundo os níveis de insegurança alimentar, escolaridade materna e faixa etária de crianças de 6 a 59 meses de idade assistidas em unidades básicas de saúde, Município do Rio de Janeiro, Brasil, 2014.

\begin{tabular}{|c|c|c|c|c|c|c|}
\hline Variáveis & $\begin{array}{c}\text { Média de } \\
\text { hemoglobina }\end{array}$ & $\begin{array}{l}\text { Desvio } \\
\text { padrão }\end{array}$ & Valor de $p$ * & $\begin{array}{l}\text { Média de } \\
\text { retinol }\end{array}$ & $\begin{array}{l}\text { Desvio } \\
\text { padrão }\end{array}$ & Valor de p * \\
\hline Nível de insegurança alimentar & & & 0,887 & & & 0,268 \\
\hline Segurança alimentar & 11,97 & 0,07 & & 1,01 & 0,02 & \\
\hline Insegurança alimentar leve & 11,92 & 0,08 & & 0,96 & 0,02 & \\
\hline Insegurança alimentar moderada & 11,83 & 0,20 & & 0,96 & 0,07 & \\
\hline Insegurança alimentar grave & 11,85 & 0,22 & & 0,82 & 0,09 & \\
\hline Escolaridade materna & & & 0,077 & & & 0,035 \\
\hline Ensino Fundamental Incompleto & 11,90 & 0,10 & & 0,93 & 0,03 & \\
\hline Ensino Fundamental Completo & 11,76 & 0,08 & & 0,97 & 0,02 & \\
\hline Ensino Médio & 11,99 & 0,08 & & 1,02 & 0,02 & \\
\hline Ensino Superior & 12,54 & 0,30 & & 1,09 & 0,06 & \\
\hline \multicolumn{7}{|l|}{ Faixa etária (meses) } \\
\hline $6-23$ & 11,54 & 0,10 & $<0,001$ & 1,02 & 0,02 & 0,163 \\
\hline $24-59$ & 12,09 & 0,06 & & 0,97 & 0,01 & \\
\hline
\end{tabular}

* Teste F ANOVA.

categorizações (segurança alimentar e todos os níveis de insegurança agrupados; segurança alimentar, insegurança leve, e os níveis de insegurança moderada e grave agrupados). Entretanto, os resultados não foram estatisticamente significativos e não serão apresentados nesta publicação.

\section{Discussão}

Em um contexto de prevalências de anemia e deficiência de vitamina $\mathrm{A}$ inferiores às observadas em 2006 no Brasil 4, observamos que crianças residentes em domicílios com insegurança alimentar leve apresentaram níveis mais baixos de retinol quando comparadas às moradoras de domicílios com segurança alimentar, não tendo sido observada associação estatisticamente significativa entre insegurança alimentar moderada ou grave e os níveis de retinol. Esse fato pode ser explicado, em parte, pela baixa prevalência de insegurança alimentar grave, o que pode prejudicar a identificação de uma possível associação devido à falta de poder estatístico. Adicionalmente, não foi observada associação estatisticamente significativa entre insegurança alimentar e os níveis de hemoglobina, embora as estimativas pontuais apontem para valores mais baixos deste biomarcador em crianças expostas à situação de insegurança alimentar. Da mesma maneira, o pequeno tamanho amostral nos estratos de insegurança alimentar moderada e grave pode ter influenciado a capacidade do estudo em identificar essas associações.

Uma justificativa para os achados relacionados à deficiência de vitamina $\mathrm{A}$ em crianças com insegurança alimentar é que indivíduos expostos a ambientes de privação social e econômica são mais suscetíveis à deficiência deste micronutriente, visto que, nestes cenários, dietas cronicamente pobres em vitamina A coexistem com infecções graves, saneamento inadequado e acesso insuficiente a serviços de saúde 13,38. Destaca-se que outros estudos nacionais não encontraram associação estatisticamente significativa entre retinol sérico e insegurança alimentar 18,19.

Em que pesem as diferenças em relação às características das cidades onde os trabalhos foram realizados, alguns estudos brasileiros com crianças nessa faixa etária também não encontraram associação estatisticamente significativa entre insegurança alimentar e os níveis de hemoglobina, seja esta analisada na sua forma contínua, com modelos de regressão linear, ou categórica, em modelos logísticos 18,19,20,22. O fato de outros autores não encontrarem associação entre insegurança alimentar 


\section{Figura 1}

Coeficientes de regressão quantílica para a associação entre insegurança alimentar e níveis de retinol e hemoglobina, em crianças de 6 a 59 meses assistidas em unidades básicas de saúde, Município do Rio de Janeiro, Brasil, 2014.
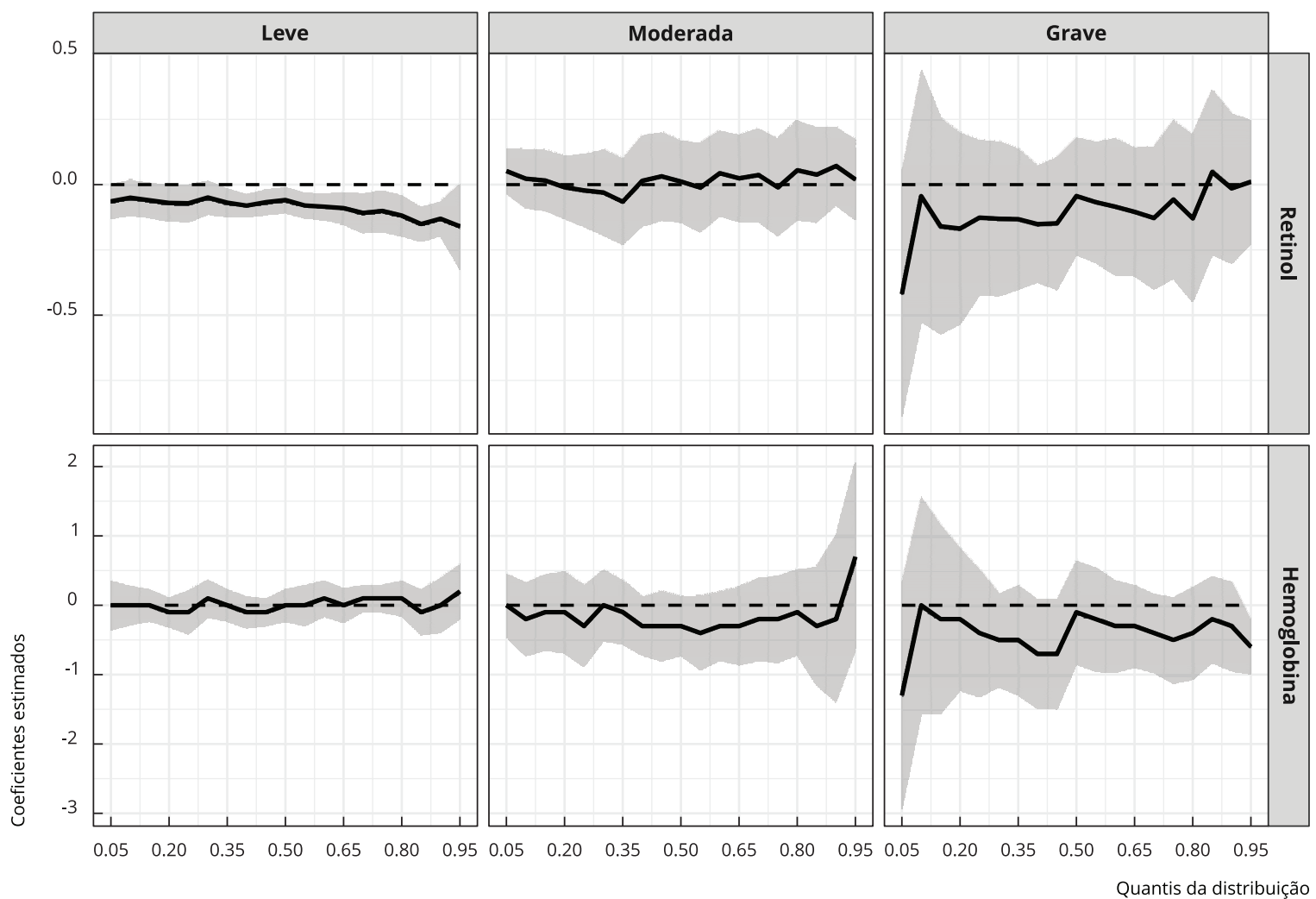

Notas: categoria de referência: segurança alimentar.

Modelo para retinol: ajustado pela escolaridade materna e PCR.

Modelo para hemoglobina: ajustado pela faixa etária da criança.

A linha cheia representa os coeficientes da regressão para os diferentes quantis da distribuição de retinol e hemoglobina, os quais foram calculados do 5 o ao 95 o quantil, com intervalos de cinco quantis.

A área cinza corresponde ao intervalo de $95 \%$ de confiança da estimativa em cada quantil. Nas situações em que o valor zero está incluído, a associação naquele quantil da distribuição não foi significativa.

e carências de micronutrientes pode ser devido ao local de realização dos estudos conduzidos até o momento no país, uma vez que insegurança alimentar é muito prevalente nas populações investigadas, o que torna os grupos analisados mais homogêneos e dificulta a identificação de uma possível associação 19. Da mesma forma, os níveis de hemoglobina e retinol variaram pouco na população estudada, o que também pode dificultar a aferição de uma associação estatisticamente significativa.

Embora o presente trabalho não tenha analisado o consumo alimentar, considerando-se que a causa mais proximal das carências de ferro e vitamina A está ligada à dieta (alimentos-fonte e fatores que influenciam a biodisponibilidade), cabe comentar estudos que tenham examinado a associação entre insegurança alimentar e a deficiência de micronutrientes levando em conta também os alimentos consumidos. Utilizando dados da PNDS-2006, pesquisa de base populacional com representatividade nacional e das macrorregiões, Araújo (2013, apud Pivetti 17) avaliou a associação entre insegurança alimentar e anemia em mães e filhos e observou que, entre as mães, a anemia foi mais frequente para aquelas em condições socioeconômicas desfavoráveis e em situação de insegurança alimentar. 
Já entre as crianças, a doença estava relacionada à idade, ao consumo alimentar e ao estado nutricional, não sendo encontrada associação entre anemia e insegurança alimentar. A autora sugere que, entre as mães, as restrições alimentares ocasionadas por condições socioeconômicas tiveram maior impacto; já entre as crianças, independentemente da condição socioeconômica, uma alimentação inadequada as torna mais suscetíveis à anemia.

Apesar de nem todas as pesquisas apontarem para a mesma direção ${ }^{39}$, a relação entre consumo alimentar e insegurança alimentar foi evidenciada em alguns estudos. Ao analisar o consumo alimentar de crianças em uma região de alta prevalência de insegurança alimentar domiciliar no Rio de Janeiro, Antunes et al. 21 apontaram que o percentual de inadequação da ingestão de proteínas e de ferro foi maior entre crianças com algum grau de insegurança alimentar quando comparadas àquelas que estavam em situação de segurança alimentar. Bortolini et al. 40, utilizando dados da PNDS-2006, também observaram piora na qualidade e diversidade da dieta em crianças que residiam em domicílios em situação de insegurança alimentar grave. Ainda que a insegurança alimentar pareça influenciar a alimentação das crianças, devido ao desenho seccional dos estudos (incluindo este), não é possível garantir que o menor consumo de nutrientes, possivelmente acarretado pela condição de insegurança alimentar, já tenha produzido a situação biológica de deficiência nutricional.

A magnitude da insegurança alimentar observada no presente estudo (40\%), apesar de ser alta, é similar à verificada em domicílios com crianças menores de cinco anos na Região Sudeste (38,3\%) e menor do que a estimativa nacional (45,5\%) em $2006^{3}$. Em creches públicas na cidade de Taubaté, São Paulo, no ano de 2014, a magnitude também foi similar, porém a prevalência de insegurança alimentar grave foi mais alta $(12,7 \%) 22$. Em alguns municípios do país, outros trabalhos mostraram prevalências em torno de $70 \%$ 21. Por outro lado, em nosso estudo, as frequências para as formas moderada e grave (3,9 e 3,1\%, respectivamente) são compatíveis com os valores encontrados nas regiões Sul e Sudeste em 2006 ( $5 \%$ e 2,8\%, $8 \%$ e 2,4\%, respectivamente) e bem menores do que as observadas na Região Nordeste no mesmo ano (21\% e 8,4\%, respectivamente) ${ }^{3}$. Esses dados mostram que a população deste trabalho, mesmo sendo assistida pelo SUS e em sua maioria com baixa renda, apresentava baixas prevalências de insegurança alimentar em suas formas moderada e grave no ano de 2014.

Uma limitação do presente estudo é a ausência de informações sobre o consumo alimentar das crianças. Outra é o tamanho da amostra, especialmente nas categorias de insegurança alimentar moderada $(n=20)$ e grave $(n=14)$, o que, como dito anteriormente, pode ter reduzido o poder do trabalho de detectar possíveis associações destes níveis de insegurança com os desfechos estudados. Uma vez que a intenção do estudo é analisar uma relação supostamente causal, a amostra deve ter tamanho suficiente para que ambos os fenômenos (desfecho e exposição em seus diferentes níveis) ocorram naquele contexto. Dessa forma, haverá um número suficiente de indivíduos em cada grupo de comparação e será possível, por meio das análises estatísticas, captar essas diferenças entre grupos 41,42. Ainda assim, os resultados aqui apresentados fornecem evidências que sugerem associação inversa entre níveis de hemoglobina e retinol sérico e níveis de insegurança alimentar.

Como pontos fortes, destacamos que este estudo foi conduzido em uma amostra representativa de crianças usuárias do SUS no Município do Rio de Janeiro e que o método utilizado para avaliar os marcadores bioquímicos foi o considerado de referência. Além disso, a associação entre insegurança alimentar e níveis de hemoglobina e retinol foi investigada por meio de modelos de regressão quantílica, tendo em vista que as relações entre os níveis de insegurança alimentar e as concentrações de retinol e hemoglobina não foram lineares. Dessa forma, introduziu-se na literatura uma nova abordagem para o estudo dessa associação. Ressalta-se, ainda, que não existiam estimativas representativas do município, nem mesmo do Estado do Rio de Janeiro, sobre os fenômenos estudados em usuários do SUS. O presente trabalho traz essa contribuição para possíveis investigações futuras.

Os resultados do estudo revelaram uma associação inversa, estatisticamente significativa, entre insegurança alimentar leve e níveis de retinol. Ainda que sem significância estatística, possivelmente devido ao pequeno tamanho amostral nesses estratos, os achados sugerem associação entre os níveis de insegurança alimentar moderada e grave e níveis de hemoglobina, e entre insegurança alimentar grave e níveis de retinol. Diante do exposto, mais investigações são necessárias nessa temática, até mesmo para compreender essas relações diante das atuais medidas de austeridade econômica e de desmonte das políticas de garantias de direitos, que podem afetar a saúde infantil 43,44. 


\section{Colaboradores}

L. B. V. Carneiro colaborou na coleta e processamento dos dados, análise, interpretação e discussão dos resultados, redação e revisão do artigo. I. R. R. Castro e L. O. Cardoso colaboraram no planejamento e coordenação da pesquisa, na interpretação e discussão dos resultados, redação e revisão do artigo. L. L. Juvanhol colaborou na análise, interpretação e discussão dos resultados e revisão do artigo. F. S. Gomes colaborou no planejamento do estudo, na análise e discussão dos resultados, e revisão do artigo.

\section{Informações adicionais}

ORCID: Letícia Barroso Vertulli Carneiro (00000003-0832-2293); Inês Rugani Ribeiro de Castro (0000-0002-7479-4400); Leidjaira Lopes Juvanhol (0000-0001-8012-6006); Fabio da Silva Gomes (0000-0003-4997-4642); Letícia de Oliveira Cardoso (0000-0003-1312-1808).

\section{Referências}

1. Food and Agriculture Organization of the United Nations; International Fund for Agricultural Development; World Food Programme. The state of food insecurity in the world: strengthening the enabling environment for food security and nutrition. Rome: Food and Agriculture Organization of the United Nations; 2014.

2. Brasil. Lei no 11.346 , de 15 de setembro de 2006. Cria o Sistema Nacional de Segurança Alimentar e Nutricional - SISAN com vistas em assegurar o direito humano à alimentação adequada e dá outras providências. Diário Oficial da União 2006; 18 set.

3. Poblacion AP, Marín-León L, Segall-Corrêa AM, Silveira JA, Taddei JAAC. Insegurança alimentar em domicílios brasileiros com crianças menores de cinco anos. Cad Saúde Pública 2014; 30:1067-78.

4. Ministério da Saúde. Pesquisa Nacional de Demografia e Saúde da Criança e da Mulher PNDS 2006: dimensões do processo reprodutivo e da saúde da criança. Brasília: Ministério da Saúde; 2009.

5. Instituto Brasileiro de Geografia e Estatística. Pesquisa Nacional por Amostra de Domicílios: segurança alimentar 2004-2009. Rio de Janeiro: Instituto Brasileiro de Geografia e Estatística; 2010.

\section{Agradecimentos}

Agradeço a toda equipe que participou do estudo e principalmente às nutricionistas Juliana Martins Oliveira e Patricia Afonso Maia. Ao CNPq, Fiocruz, Faperj e Prefeitura da Cidade do Rio de Janeiro.

\section{Conflito de interesses}

F. S. Gomes é funcionário da Organização Pan-Americana da Saúde (OPAS), e o conteúdo deste artigo é de responsabilidade dos autores e não necessariamente reflete os critérios ou a política da OPAS. Em qualquer uso desta publicação, não deve haver nenhuma sugestão de que a OPAS endossa qualquer organização, produto ou serviço específico.
6. Instituto Brasileiro de Geografia e Estatística. Pesquisa Nacional por Amostra de Domicílios - pesquisa suplementar de segurança alimentar 2013. Rio de Janeiro: Instituto Brasileiro de Geografia e Estatística; 2014.

7. Recine E, Beghin N. A agenda de nutrição das estratégias internacionais: iniciativas em andamento, desafios e propostas. Contribuições para debate no Conselho Nacional de Segurança Alimentar e Nutricional - CONSEA. Brasília: Conselho Nacional de Segurança Alimentar e Nutricional; 2014.

8. Departamento de Atenção Básica, Secretaria de Atenção à Saúde, Ministério da Saúde. Política nacional de alimentação e nutrição. Brasília: Ministério da Saúde; 2012.

9. Monteiro CA, Moubarac JC, Cannon G, Ng SW, Popkin B. Ultra-processed products are becoming dominant in the global food system. Obes Rev 2013; 14 Suppl 2:21-8.

10. Martins AP, Levy RB, Claro RM, Moubarac JC, Monteiro CA. Participação crescente de produtos ultraprocessados na dieta brasileira (19872009). Rev Saúde Pública 2013; 47:656-65.

11. Louzada MLC, Martins APB, Canella DS, Baraldi LG, Levy RB, Claro RM, et al. Impacto de alimentos ultraprocessados sobre o teor de micronutrientes da dieta no Brasil. Rev Saúde Pública 2015; 49:45. 
12. United Nations Children's Fund. Preventing iron deficiency in women and children: background and consensus on key technical issues and resources for advocacy, planning and implementing national programmes. New York: United Nations Children's Fund; 1998. (Technical Workshop Section, 7).

13. World Health Organization. Global prevalence of vitamin A deficiency in populations at risk 1995-2005. WHO global database on vitamin A deficiency. Geneva: World Health Organization; 2009.

14. Ramalho RA, Flores H, Accioly E, Saunders C. Associação entre deficiência de vitamina A e situação sociodemográfica de mães e recémnascidos. Rev Assoc Med Bras 2006; 52:170-5.

15. Santos EN, Velarde LGC, Ferreira VA. Associação entre deficiência de vitamina A e variáveis socioeconômicas, nutricionais e obstétricas de gestantes. Ciênc Saúde Colet 2010; 15:1021-30.

16. André HP, Sperandio N, Siqueira RL, Franceschini SCC, Priore SE. Food and nutrition insecurity indicators associated with iron deficiency anemia in Brazilian children: a systematic review. Ciênc Saúde Colet 2018; 1159-67.

17. Pivetti F. Ambiente familiar influi em casos de anemia em mães e filhos, aponta estudo da EE. de São Paulo. Agência USP de Notícias 2013; 1 abr. https://www5.usp.br/24655/ambiente-fa miliar-influi-em-casos-de-anemia-em-maes-efilhos-aponta-estudo-da-ee/.

18. Pedraza DF, Queiroz D, Paiva AA, Cunha MAL, Lima ZN. Seguridad alimentaria, crecimiento y niveles de vitamina $\mathrm{A}$, hemoglobina y zinc en niños preescolares del nordeste de Brasil. Ciênc Saúde Colet 2014; 19:641-50.

19. Oliveira JS, Lira PIC, Osório MM, Sequeira LAS, Costa EC, Gonçalves FCLSP, et al. Anemia, hipovitaminose A e insegurança alimentar em crianças de municípios de baixo índice de desenvolvimento humano do nordeste do Brasil. Rev Bras Epidemiol 2010; 13:651-64.

20. Gubert MB, Spaniol AM, Bortolini GA, PérezEscamilla R. Household food insecurity, nutritional status and morbidity in Brazilian children. Public Health Nutr 2016; 19:2240-5.

21. Antunes MML, Sichieri R, Salles-Costa R. Consumo alimentar de crianças menores de três anos residentes em área de alta prevalência de insegurança alimentar domiciliar. Cad Saúde Pública 2010; 26:1642-50.

22. Rocha EMB, Abreu LC, Lopes AF, Leone C, Vieira PD, Bezerra IMP, et al. Relation of food insecurity and hemoglobin level in preschool aged children. Anemia 2018; 2018:1-7.

23. Shamah Levy T, Méndez-Gómez-Humarán I, Morales Ruán MD, Martinez Tapia B, Villalpando Hernández S, Hernández Ávila M. Validation of masimo pronto 7 and HemoCue 201 for hemoglobin determination on children from 1 to 5 years of age. PLoS One 2017; 12:e0170990.
24. Hinnouho GM, Barffour M, Wessells KR, Brown KH, Kounnavong S, Chanhthavong B, et al. Comparison of haemoglobin assessments by HemoCue and two automated haematology analysers in young Laotian children. J Clin Pathol 2018; 71:532-8.

25. Gwetu TP, Chhagan K. Evaluation of the diagnostic accuracy of the HemoCue device for detecting anaemia in healthy schoolaged children in KwaZulu-Natal, South Africa. S Afr Med J 2015; 105:596-9.

26. Departamento de Atenção Básica, Secretaria de Atenção à Saúde, Ministério da Saúde, Fundo das Nações Unidas para a Infância. Carências de micronutrientes. Brasília: Ministério da Saúde; 2007. (Cadernos de Atenção Básica 20) (Série A. Normas e Manuais Técnicos).

27. World Health Organization. Guideline: intermittent iron supplementation in preschool and school-age children. Geneva: World Health Organization; 2011.

28. World Health Organization. Guideline: vitamin A supplementation in infants and children 6-59 months of age. Geneva: World Health Organization; 2011.

29. Vieira RCS, Ferreira HS. Prevalence of anemia in Brazilian children in different epidemiological scenarios. Rev Nutr 2010; 23:433-44.

30. Pérez-Escamilla R, Segall-Corrêa AM, Kurdian Maranha G, Sampaio MMF, Marin-Leon L, Panigassi L. An adapted version of the US Department of Agriculture Food Insecure Module is a valid tool for assessing food insecurity in Campinas, Brasil. J Nutr 2004; 134:1923-8.

31. Comissão de Coleta de Sangue Venoso, Medicina Laboratorial, Sociedade Brasileira de Patologia Clínica. Recomendações da Sociedade Brasileira de Patologia Clínica Medicina Laboratorial para coleta de sangue venoso. 2 a Ed. Barueri: Minha Editora; 2010.

32. Hess D, Keller HE, Oberlin B, Bonfanti R, Schüep W. Simultaneous determination of retinol, tocopherols, carotenes and lycopene in plasma by means of high-performance liquid chromatography on reversed phase. Int J Vitam Nutr Res 1991; 61:232-8.

33. Craft NE, Haitema T, Brindle LK, Yamini S, Humphrey JH, West Jr. KP. Retinol analysis in dried blood spots by HPLC. J Nutr 2000; 130:882-5.

34. World Health Organization. Iron deficiency anemia: assessment, prevention and control - a guide for programme managers. Geneva: World Health Organization; 2001.

35. Buchinsky M. Recent advancens in quantile regression models: a practical guideline for empirical research. J Hum Resour 1998; 33:88126.

36. Koenker R. Quantile regression. New York: Cambridge University Press; 2005. 
37. Beyerlein A. Quantile regression - opportunities and challenges from a user's perspective. Am J Epidemiol 2014; 180:330-1.

38. Ramalho RA, Flores H, Saunders C. Hipovitaminose A no Brasil: um problema de saúde pública. Rev Panam Salud Pública 2002; 12:11722.

39. Ruschel LF, Henn RL, Backes V, Melo P, Marques LAS, Olinto MTA. Insegurança alimentar e consumo alimentar inadequado em escolares da rede municipal de São Leopoldo, RS, Brasil. Ciênc Saúde Colet 2016; 21:2275-85.

40. Bortolini GA, Vitolo MR, Gubert MB, Santos LMP. Iniquidades sociais influenciam a qualidade e a diversidade da dieta de crianças brasileiras de 6 a 36 meses. Cad Saúde Pública 2015; 31:2413-24.

41. Altman DG. Pratical statistics for medical research. London: Chapman \& Hall; 1991.
42. Almeida Filho N, Barreto ML. Epidemiologia \& Saúde: fundamentos, métodos e aplicações. Rio de Janeiro: Guanabara Koogan; 2012.

43. Rasella D, Basu S, Hone T, Paes-Sousa R, Ocké-Reis CO, Millett C. Child morbidity and mortality associated with alternative policy responses to the economic crisis in Brazil: a nationwide microsimulation study. PLoS Med 2018; 15:e1002570.

44. Santarelli M, Burity V, Silva LNB, Prates L, Rizzolo A, Rocha NC, et al. Da democratização ao golpe: avanços e retrocessos na garantia do direito humano à alimentação e à nutrição adequadas no Brasil. Brasília: Organização pelo Direito Humano à Alimentação e à Nutrição Adequadas; 2017. 


\section{Abstract}

This study analyzed the association between food insecurity and hemoglobin and retinol levels in children 6 to 59 months of age. This was a crosssectional study in 2014 with a representative sample of children in this age bracket treated at basic health units in the city of Rio de Janeiro, Brazil. Analysis of food insecurity levels used the Brazilian Food Insecurity Scale, and venipuncture was performed for measurement of serum hemoglobin and retinol levels. The association between variables used quantile regression models. Of all the children in the sample, $40.3 \%$ presented food insecurity, and the prevalence rates for anemia and vitamin A deficiency were $13.7 \%$ and $13 \%$, respectively. The study's results revealed a statistically significant inverse association between mild food insecurity and retinol levels. For the other levels of food insecurity (moderate and severe), the results also suggest an inverse association for hemoglobin, and for retinol levels the point estimates appear smaller in children with severe food insecurity, but these estimates were not statistically significant. These results suggest that food insecurity may be associated with micronutrient deficiencies in children under 5 years.

Food and Nutyrition Security; Anemia; Vitamin A Deficiency; Child

\section{Resumen}

En este estudio se analizó la asociación entre la inseguridad alimentaria y los niveles de hemoglobina y retinol en niños de 6 a 59 meses de edad. Se trata de un estudio seccional, realizado en 2014, con una muestra representativa de la población de niños en esta franja etaria, atendida en unidades básicas de salud del Municipio de Río de Janeiro, Brasil. Para el análisis de los niveles de inseguridad alimentaria se utilizó la Escala Brasileña de Inseguridad Alimentaria $y$, para la determinación de hemoglobina y de retinol sérico, se realizó una punción venosa. La asociación entre las variables se evaluó a través de modelos de regresión cuantílica. Del total de niños estudiados, un 40,3\% presentaban inseguridad alimentaria y las prevalencias de anemia y de deficiencia de vitamina $A$ fueron $13,7 \%$ y $13 \%$, respectivamente. Los resultados del estudio revelaron una asociación inversa, estadísticamente significativa, entre inseguridad alimentaria leve y niveles de retinol. Para los demás niveles de inseguridad alimentaria (moderada y grave), los resultados también sugieren la presencia de una asociación inversa para la hemoglobina, y, en cuanto a los niveles de retinol, las estimaciones puntuales parecen menores en niños con inseguridad alimentaria grave, sin embargo, estas estimaciones no fueron estadísticamente significativas. Estos resultados sugieren que la inseguridad alimentaria puede estar asociada con carencias de micronutrientes en niños menores de 5 años.

Seguridad Alimentaria y Nutricional; Anemia; Deficiencia de Vitamina A; Niño
Recebido em 18/Dez/2018

Versão final reapresentada em 09/Mai/2019 Aprovado em 20/Mai/2019 